\title{
A Characterization of the Diffuse Galactic Emissions at Large Angular Scales Using the Tenerife Data
}

\author{
J. F. Macías-Pérez, ${ }^{1,2}$ R. D. Davies, ${ }^{2}$ R. Watson, ${ }^{2}$ C. M. Gutierrez, ${ }^{3}$ and R. Rebolo ${ }^{3}$ \\ ${ }^{1}$ LPSC, Université Joseph Fourier Grenoble 1, CNRS/IN2P3, Institut National Polytechnique de Grenoble, 53 avenue des Martyrs, \\ 38026 Grenoble Cedex, France \\ ${ }^{2}$ Jodrell Bank Centre for Astrophysics, Alan Turing Building, School of Physics and Astronomy, The University of Manchester, \\ Oxford Road, Manchester M13 9PL, UK \\ ${ }^{3}$ Instituto de Astrofísica de Canarias, C/Vía Láctea s/n, La Laguna, 65 Tenerife, Spain \\ Correspondence should be addressed to J. F. Macías-Pérez; macias@lpsc.in2p3.fr
}

Received 9 July 2012; Revised 13 January 2013; Accepted 13 January 2013

Academic Editor: Roberta Paladini

Copyright ( 2013 J. F. Macías-Pérez et al. This is an open access article distributed under the Creative Commons Attribution License, which permits unrestricted use, distribution, and reproduction in any medium, provided the original work is properly cited.

\begin{abstract}
The anomalous microwave emission (AME) has been proved to be an important component of the galactic diffuse emission in the range from 20 to $60 \mathrm{GHz}$. To discriminate between different models of AME, low frequency microwave data from 10 to $20 \mathrm{GHz}$ are needed. We present here a reanalysis of published and unpublished Tenerife data from 10 to $33 \mathrm{GHz}$ at large angular scales (from 5 to 15 degrees). We cross-correlate the Tenerife data to templates of the main galactic diffuse emissions: synchrotron, free-free, and thermal dust. We find evidence of dust-correlated emission in the Tenerife data that could be explained as spinning dust grain emission.
\end{abstract}

\section{Introduction}

The anomalous microwave emission (AME) is an important contributor of the galactic diffuse emission in the range from 20 to $60 \mathrm{GHz}$. It was first identified by $[1,2]$ as freefree emission from electrons with temperature, $T_{e}>10^{6} \mathrm{~K}$. Draine and Lazarian [3] argued that AME may result from electric dipole radiation due to small rotating grains, the socalled spinning dust. Models of the spinning dust emission, Draine and Lazarian [4] show that an emissivity spectrum peaking at around $20-50 \mathrm{GHz}$ is able to reproduce the observations [5-12]. The initial spinning dust model has been refined regarding the shape and rotational properties of the dust grains [13-15]. An alternative explanation of AME was proposed by Draine and Lazarian [16] based on magnetic dipole radiation arising from hot ferromagnetic grains. Observations have placed limits of a few percent on the fractional polarization towards AME targets $[9,17-$ 20]. This excludes perfectly aligned single-domain magnetic grains; however, other alignments and grain compositions produce similarly low levels of polarization [21].
A correlation between microwave and infrared maps, mainly dominated by dust thermal emission [22], was observed for various experiments, for example, on COBE/DMR [23, 24], OVRO [1, 2], Saskatoon [25, 26], survey at $19 \mathrm{GHz}[27,28]$, and Tenerife $[29,30]$. A similar signal was found in compact regions by [5] and in some molecular clouds based on data from COSMOSOMAS [7, 31], AMI (Ami-Consortium: [32, 33]), CBI [9, 34], VSA [12], and Planck [35]. A recent study of the Small Magellanic Cloud also claims a detection of AME [36].

Independently, Bennett et al. [41] proposed an alternative explanation of AME based on flat-spectrum synchrotron emission associated to star-forming regions to explain part of the WMAP first-year observations. This hypothesis seems to be in disagreement with results from de Oliveira-Costa et al. [6]; Fernández-Cerezo et al. [42]; Hildebrandt et al. [43]; Ysard et al. [44] which showed that spinning dust best explained the excess below $20 \mathrm{GHz}$. Furthermore, Davies et al. [45] showed the existence of important correlation between microwave and infrared emission in regions outside star-forming areas. More recently, Kogut et al. [46] discussed 
the fact that spinning dust fits better to ARCADE data (3.8 and $10 \mathrm{GHz}$ ) than a flat-spectrum synchrotron.

To discriminate between the different AME models and the alternative explanations such as those discussed earlier low frequency microwave data in the range, from 10 to $20 \mathrm{GHz}$ at different angular resolutions are needed. Indeed in this frequency range, we expect the AME spectrum to be significantly distinct from magnetic dust and flat-spectrum synchrotron. At this respect, the Tenerife data set, from 10 to $33 \mathrm{GHz}$ and at large angular scales (from 5 to 15 degrees), is unique. We present in the following a re-analysis of these data including previously published data by Gutierrez et al. [47] and un-published data since January 1998 to December 2000. The paper is structured as follows. Section 2 presents the Tenerife data and discusses the reprocessing of these data. In Section 3 we describe the main galactic emission mechanisms and the associated templates used in the analysis. Section 4 discusses the point source contribution to the Tenerife data. In Section 5, we present the cross correlation between the Tenerife data and the galactic templates. The main results are discussed in Section 6. Finally, we draw conclusions in Section 7.

\section{The Tenerife Data}

The Tenerife experiment observed unidimensional scans at constant declination at 10,15 , and $33 \mathrm{GHz}$ using a triple beam pattern of FWHM $5^{\circ}$ and of $8.1^{\circ}$ beam spacing. The region of the sky centered at declination $40^{\circ}$ was chosen for observations because it corresponds to the largest area of the sky at high latitudes where contamination from foregrounds is a minimum. To reconstruct $2 \mathrm{D}$ maps of the sky, consecutive declinations separated by half the beamwidth $\left(2.5^{\circ}\right)$ are observed. Each single declination is repeatedly observed until sufficient sensitivity and full RA coverage is achieved. The scheduling of the observations takes into account the position of the sun so that its contribution to the data is a minimum. This requires observations of the same declination at different times of the year for full RA coverage. Observations were performed day and night. Day-time observations present an increase in total power and noise with respect to night-time observations due to receiver gain changes. In extreme cases, data observed at day time have to be removed.

The atmospheric contribution to the data depends on frequency, being severe at $33 \mathrm{GHz}$ and relatively small at $10 \mathrm{GHz}$. The observing efficiency depends mainly on the weather conditions. Data strongly affected by atmospheric contamination cannot be used. In addition, technical problems, such as power cutoff, warmed and oscillating HEMT amplifiers, RF interference, and failure in the electronic systems, can also affect the data. At 10 and $15 \mathrm{GHz}$, more than $80 \%$ of the observed data are useful. However, at $33 \mathrm{GHz}$ only about $10 \%$ of data are kept, due mainly to atmospheric effects.

We use in the following the full Tenerife data set which includes new data (taken from January 1998 to December 2000) with respect to previously published releases (see, e.g., $[47,48])$. These data have been completely reprocessed
TABLE 1: $10 \mathrm{GHz}$ data. Mean temperature, mean noise per beam, and r.m.s per $1^{\circ}$ pixel for the $10 \mathrm{GHz}$ final stacks in the RA interval $150^{\circ}$ to $250^{\circ}$.

\begin{tabular}{lccc}
\hline Declination $(\mathrm{deg})$ & Mean $T(\mu \mathrm{K})$ & $\sigma(\mu \mathrm{K})$ & r.m.s $(\mu \mathrm{K})$ \\
\hline 30.0 & -3.7 & 52.9 & 150.5 \\
32.5 & -5.0 & 45.9 & 147.8 \\
35.0 & 10.0 & 47.1 & 140.5 \\
37.5 & 7.5 & 51.0 & 153.1 \\
40.0 & 13.6 & 49.6 & 147.4 \\
42.5 & 7.9 & 54.1 & 146.0 \\
45.0 & -7.8 & 59.2 & 215.5 \\
\hline
\end{tabular}

TABLE 2: $15 \mathrm{GHz}$ data. mean, mean noise per beam, and r.m.s per $1^{\circ}$ pixel for the $15 \mathrm{GHz}$ final stacks in the RA interval $150^{\circ}$ to $250^{\circ}$.

\begin{tabular}{lccc}
\hline Declination & Mean $T(\mu \mathrm{K})$ & $\sigma(\mu \mathrm{K})$ & r.m.s $(\mu \mathrm{K})$ \\
\hline 25.0 & 3.6 & 18.1 & 51.7 \\
27.5 & 4.6 & 16.3 & 48.7 \\
30.0 & 3.4 & 19.3 & 61.1 \\
32.5 & 3.9 & 22.7 & 67.2 \\
35.0 & 0.25 & 20.6 & 74.6 \\
37.5 & 8.3 & 22.4 & 78.6 \\
40.0 & 7.8 & 20.7 & 75.7 \\
42.5 & 5.0 & 22.6 & 81.3 \\
45.0 & -8.2 & 21.3 & 75.8 \\
47.5 & -5.9 & 19.3 & 70.5 \\
\hline
\end{tabular}

TABle 3: $33 \mathrm{GHz}$ data. Mean, mean noise per beam, and r.m.s per $1^{\circ}$ pixel for the $33 \mathrm{GHz}$ final stacks in the RA interval $150^{\circ}$ to $250^{\circ}$.

\begin{tabular}{lccc}
\hline Declination & Mean $T(\mu \mathrm{K})$ & $\sigma(\mu \mathrm{K})$ & r.m.s $(\mu \mathrm{K})$ \\
\hline 40.0 & -3.1 & 27.2 & 80.8 \\
42.5 & 0.2 & 43.7 & 167.7 \\
\hline
\end{tabular}

including hand editing to remove clearly systematic contaminated regions and accurate removal of the atmospheric emission using an MEM-based baseline removal technique as described in Gutierrez et al. [47]. The latter has been improved to enlarge the sky region for which atmospheric residuals are negligible. In particular, this allows us to consider in the following analysis regions at low galactic latitudes. Figures 1, 2, and 3 present the cleaned Tenerife data at 10, 15, and $33 \mathrm{GHz}$, respectively. The main properties of these clean data: central declination of the scan, mean temperature, mean noise per beam, and r.m.s per $1^{\circ}$ pixel in the RA interval $150^{\circ}$ to $250^{\circ}$, are presented in Tables 1,2 and 3 for the 10,15 , and $33 \mathrm{GHz}$ channels, respectively.

\section{Galactic Diffuse Emission}

We present in this section the main known galactic diffuse mechanisms: synchrotron, free-free, and vibrational and rotational dust. 

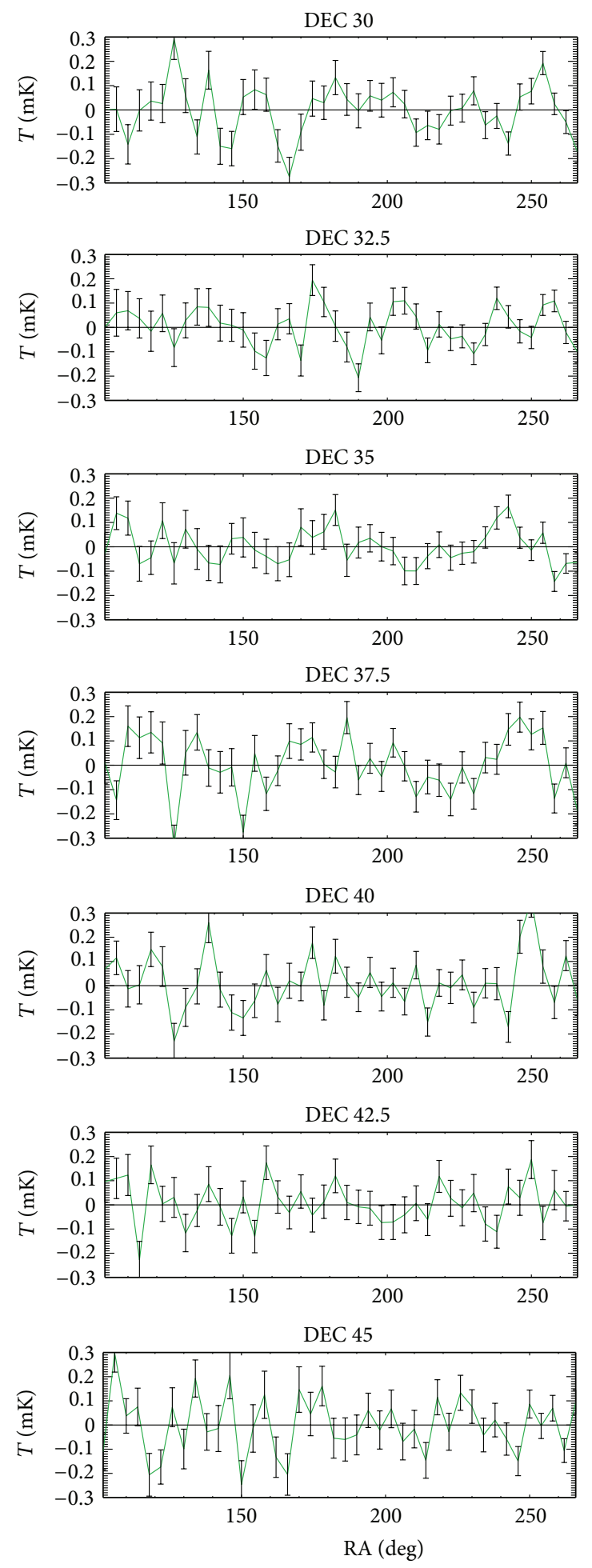

Figure 1: $10 \mathrm{GHz}$ stacked scans. From top to bottom we plot the stacked scans for the $10 \mathrm{GHz}$ radiometer from declination $30^{\circ}$ to $45^{\circ}$. The data have been binned into $4^{\circ}$ pixels.

3.1. Synchrotron. Synchrotron emission results from cosmicray electrons accelerated in magnetic fields, and thus, depends on the energy spectrum of the electrons and the intensity of the magnetic field $[49,50]$. The local energy spectrum of the electrons has been measured to be, for energies contributing to the observed radio synchrotron emission, a power law to good approximation with index from about -2.7 to -3.3 over this energy range [51]. Such an 


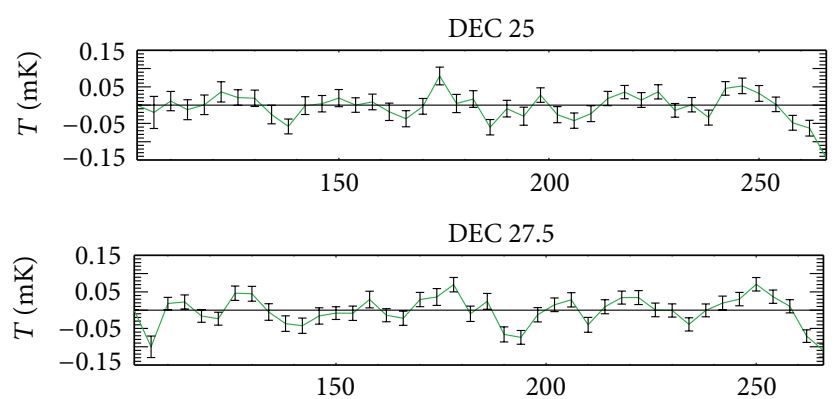

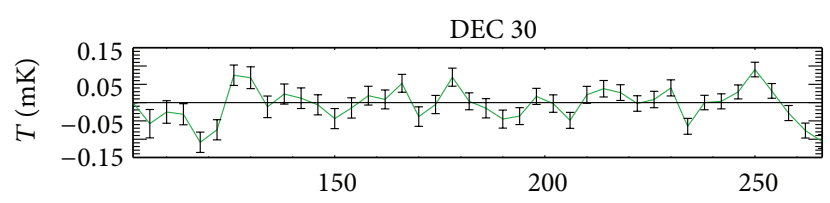

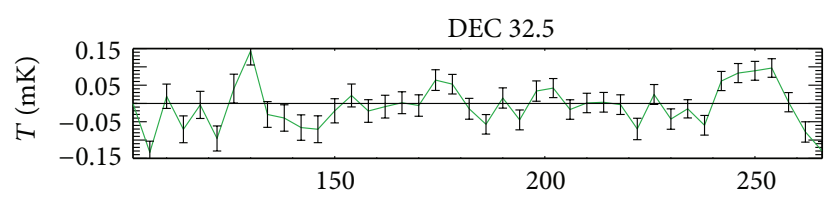

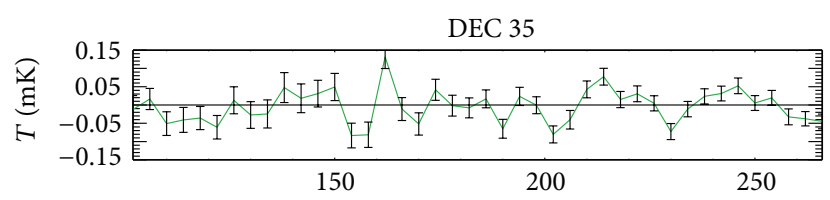

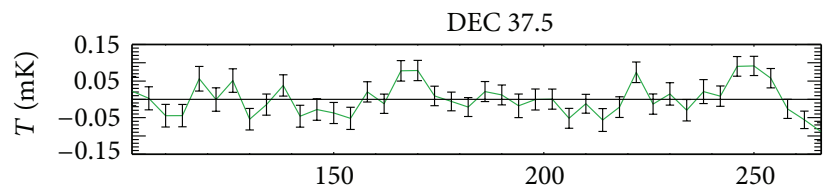

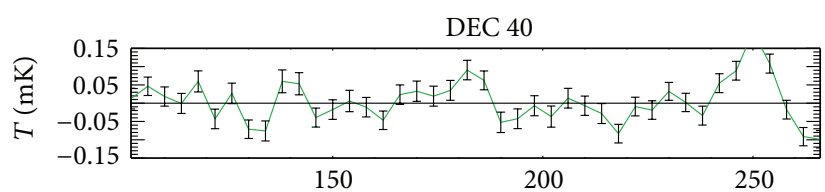

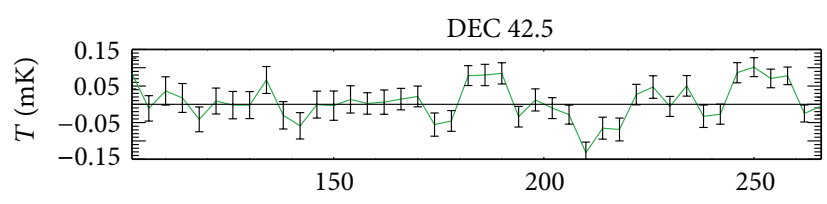

DEC 45

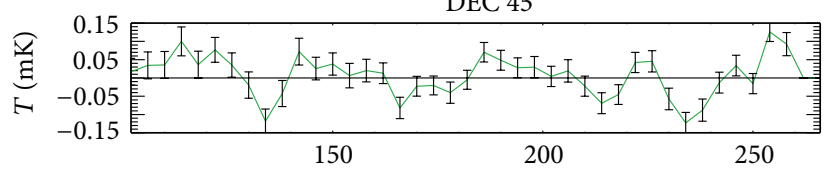

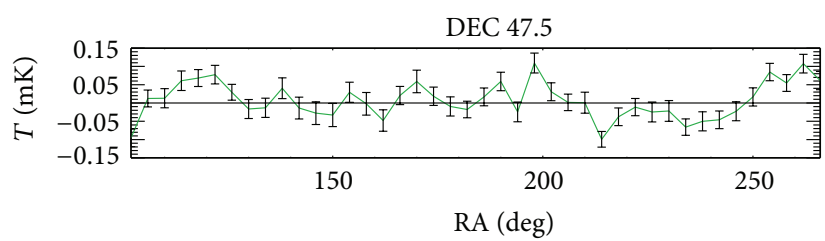

FIGURE 2: $15 \mathrm{GHz}$ stacked scans. From top to bottom we plot the $15 \mathrm{GHz}$ stacked scans in the declination range $25^{\circ}$ to $47.5^{\circ}$. The data have been binned into $4^{\circ}$ pixels. 


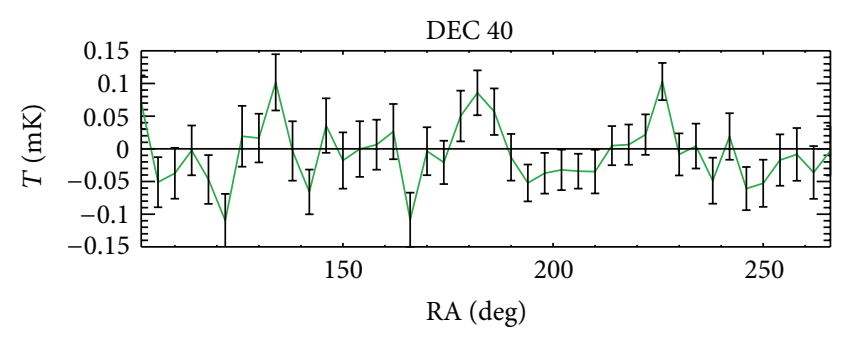

(a)

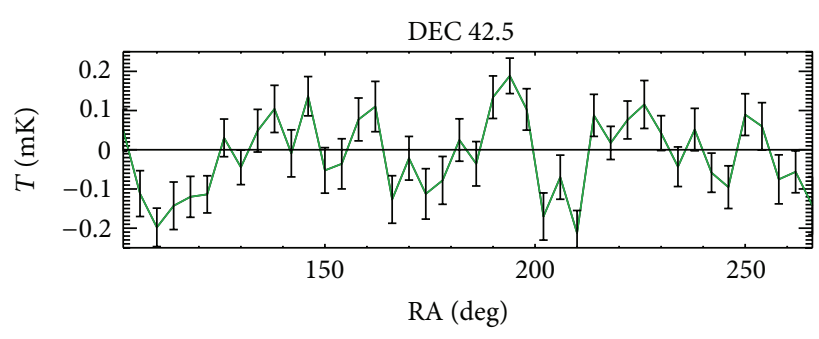

(b)

FIGURE 3: $33 \mathrm{GHz}$ stacked scans. From top to bottom we plot the stacked scans for the $33 \mathrm{GHz}$ radiometer in the triple beam configuration for declinations $40^{\circ}$ to $42.5^{\circ}$. The data have been smoothed into $4^{\circ}$ pixels.

increase of energy spectrum slope is expected, as the energy loss mechanism for electrons increases as the square of the energy.

Radio surveys at frequencies less than $2 \mathrm{GHz}$ are dominated by synchrotron emission [52]. The only all-sky survey available at these frequencies is the $408 \mathrm{MHz}$ map [53]. This survey has a resolution of $0.85^{\circ}$ and was produced using the Parkes $64 \mathrm{~m}$ telescope in Australia for the southern sky and the Bonn $100 \mathrm{~m}$ and Jodrell Bank MK1A telescopes for the northern sky. The scanning strategy with the Bonn telescope was to fix the azimuth at the local meridian and scan up and down in elevation at a rate of about $6^{\circ} / \mathrm{min}$. This technique reduced the atmospheric contribution to the map but led to a set of vertical stripes (constant RA) separated by $7^{\circ}$. The quoted errors in the temperature scale are of the order of $10 \%$ and $\pm 3 \mathrm{~K}$ in the absolute brightness temperature levels.

In addition, there is the $1420 \mathrm{MHz}$ survey [54] which covers the declination range $-19^{\circ}$ to $90^{\circ}$ and has a FWHM of $0.58^{\circ}$. Stripes are also present in this map due to the scanning strategy which consisted of azimuthal scans at constant elevation. The errors in the temperature scale are of the order of $5 \%$ and $\pm 0.5 \mathrm{~K}$ in the absolute brightness temperature.

The $408 \mathrm{MHz}$ and $1420 \mathrm{MHz}$ maps have been used to determine the synchrotron spectral index at radio frequencies $[54,55]$. Assuming that $T_{v} \propto v^{-\beta}$ and after destriping and correction for zero levels, spectral indexes, $\beta$, in the range 2.8 to 3.2 were found. The spatial angular power spectrum of the synchrotron emission $C_{\ell}^{\text {sync }}$ is poorly understood but is believed to be $C_{\ell}^{\text {sync }} \propto \ell^{-3}$ [56]. At high galactic latitudes in the region observed by the Tenerife experiment, the synchrotron spatial angular power spectrum is slightly flatter than $l^{-2}$ [57]. In the following sections, we will use the destriped version of the $408 \mathrm{MHz}$ map [55] as a template of the synchrotron emission. This map is shown in Figure 4.

3.2. Free-Free. When a charged particle is accelerated in a Coulomb field it will emit radiation which is called braking radiation or Bremsstrahlung. The galactic free-free emission is the thermal bremsstrahlung from hot electrons $\left(\sim 10^{4} \mathrm{~K}\right)$ produced in the interstellar gas by the UV radiation field [51]. This emission is not easily identified at radio frequencies, except near the galactic plane. At higher latitudes, it must be separated from synchrotron emission by virtue of their different spectral indices, since the spectral index of optically thin free-free emission is $\beta^{\mathrm{ff}}=2.1$.

The diffuse galactic recombination radiation, $H_{\alpha}$ is a good tracer of free-free emission since both are emitted in the same Warm Ionised Medium (WIM) and both have intensities proportional to the emission measure,

$$
\mathrm{EM}=\int n_{e} n_{p} d \ell \simeq \int n_{e}^{2} d \ell .
$$

The ratio of free-free brightness temperature $T_{b}^{\mathrm{ff}}$ to the $H_{\alpha}$ surface brightness $I_{\alpha}$ in $R$ (Rayleigh) is [58]

$$
\frac{T_{b}^{\mathrm{ff}}[m K]}{I_{\alpha}[R]}=10.4 v^{-2.14} T_{4}^{0.527} 10^{0.029 / T_{4}}(1+0.08),
$$

where $v$ is the observing frequency in $\mathrm{GHz}, \mathrm{T}_{4}$ is the temperature of the electrons in units of $10^{4} \mathrm{~K}$, and the last factor 0.08 corresponds to helium which is assumed completely ionised and creates free-free-emission like hydrogen but does not emit $H_{\alpha}$ light.

Recently, a full sky survey of $H_{\alpha}$ light has been released by the WHAM (Wisconsin $H_{\alpha}$ Mapper) collaboration [59]. The WHAM instrument consists of a 6 inch dual-etalon FabryPerot spectrometer with a narrow band filter of FWHM $\sim 20 \AA$ which images onto a cryogenically cooled $1024 \times$ $1024 \mathrm{CCD}$. The resolution of the survey is $\sim 1^{\circ}$. The spatial power spectrum of free-free emission $C_{\ell}^{\mathrm{ff}}$ has not yet been determined from the WHAM map; however, analysis of $H_{\alpha}$ images of the North Celestial Pole area [60] suggests that $C_{\ell}^{\mathrm{ff}} \propto \ell^{-2.27 \pm 0.07}$.

In Figure 4, we present a map of the northern sky produced by the WHAM survey. In the following sections, we will use this map as a template for the free-free emission. At intermediate Galactic latitudes (say $|b|>30^{\circ}$ ), about $10 \%$ of the $H_{\alpha}$ light is absorbed by dust and therefore estimates of free-free emission from $H_{\alpha}$ will be systematically lower than the true value. At latitudes below $10^{\circ}$, this correction becomes increasingly uncertain.

3.3. Vibrational Dust. At the higher frequency range $(\geq 100 \mathrm{GHz}$ ) of the microwave background experiments, dust emission starts to become dominant. Dust grains are heated by interstellar radiation, absorbing optical and UV photons, and emitting energy in the far infrared. 


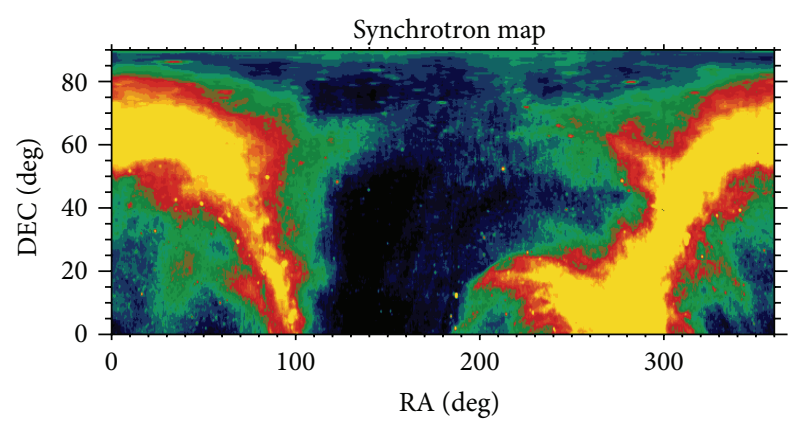

(a)

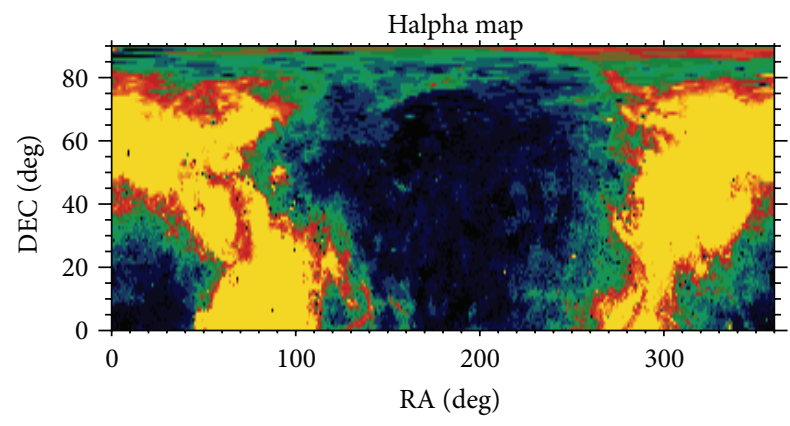

(b)

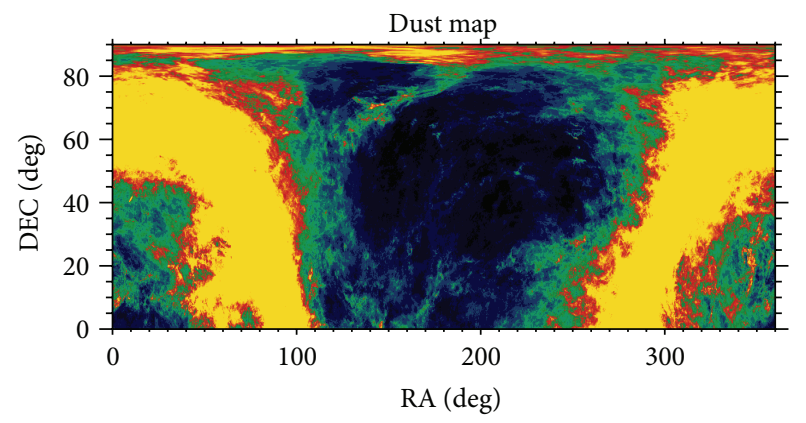

(c)

Figure 4: From top to bottom. $408 \mathrm{MHz}$ synchrotron map. Resolution: $0.85^{\circ}$ and pixels: $0.33^{\circ}$. WHAM $H_{\alpha}$ map.This map is a courtesy of the WHAM collaboration. Resolution: $1^{\circ}$ and pixels: $1^{\circ}$. Combined DIRBE plus IRAS $100 \mu \mathrm{m}$ dust map. Resolution: 6 arcmin and pixels: $0.25^{\circ}$.

The intensity of the emission from an ensemble of dust grains is given by

$$
I(v)=\int \epsilon(v) d \ell
$$

where $\epsilon(\nu)$ is the emissivity at frequency $\nu$, and the integral is along the line of sight. In the Rayleigh-Jeans regime and assuming a constant line-of-sight density of dust,

$$
T_{b} \propto \epsilon(v) v^{-2}
$$

where $T_{b}$ is the brightness temperature of the dust emission.

The spectrum of the dust emission has been measured at millimeter and submillimeter wavelengths by the Far Infrared Absolute Spectrophotometer (FIRAS) and can be fitted by a single greybody spectrum of temperature $17.5 \mathrm{~K}$ and emissivity $\propto v^{2}[61]$ at high galactic latitudes. From IRAS observations of dust emission [62], it was found that the spatial power spectrum of the dust fluctuations is $C_{\ell}^{\text {dust }} \propto$ $\ell^{-3}$. This has also been confirmed at larger angular scales by the COBE-DIRBE satellite.

In the following sections, we will use the combined IRASDIRBE map at $100 \mu \mathrm{m}$ [63] as a template for the dust emission. This map has a resolution of FWHM $\sim 6$ arcmin and covers the full sky. Zodiacal light and point sources have been removed from the map, and the regions of the sky which were not observed by the IRAS satellite have been replaced by DIRBE data. The combined map preserves the DIRBE zero point and calibration. This map is in units of MJy/sr. In Figure 4, we present the northern sky part of the combined IRAS-DIRBE map.

3.4. Rotational Dust. Small spinning interstellar dust grains containing $10^{2}-10^{3}$ atoms can produce detectable rotational emission in the $10-100 \mathrm{GHz}$ range. This emission depends on the component of the electric dipole moment perpendicular to the angular velocity of the grain and on the physical properties of the interstellar medium [3]. For these small grains, rotational excitation is dominated by direct collisions with ions and plasma drag. The very smallest grains $(N \leq$ 150) have their rotation damped primarily by electric dipole emission; for $150 \leq N \leq 10^{3}$ plasma drag dominates.

In the following sections, we show models for the spectrum of the spinning dust emission provided by Dr. Draine (private communication). These models depend on a large set of parameters such as the distribution of grain sizes, the charge of the grains, the composition of the grains, and the physical properties of the interstellar medium which were fixed by the authors. However, the normalization of the model can be assumed as a free parameter although it is related to the hydrogen column density in the interstellar medium which is of the order of a few times $10^{20}$.

The spatial distribution of small dust grains is not wellknown although it seems reasonable to believe that it is not different from that of larger grains but for dense regions where dust coagulation may deplete small grains. For the purpose of this work, we will use the IRAS-DIRBE $100 \mu \mathrm{m}$ map as a template for the spinning dust grains.

\section{Galactic and Extragalactic Point Sources}

The contribution from resolved point sources to the Tenerife data at 10 and $15 \mathrm{GHz}$ was extrapolated from data of the Michigan monitoring program (M. Aller and H. Aller 2000, private communication). The Michigan program regularly monitors point sources with fluxes above $0.5 \mathrm{Jy}$ at $4.8,8.0$, and 14.5 GHz. The Michigan catalog is neither complete in flux nor time domain. Well-known strong variable sources such as 3C345 are regularly observed, and data at all observing frequencies are available. Weak sources are poorly observed, and often data are available at a single frequency of the three possible. Further, data are available up to June 1999 while the Tenerife experiment operated until September 2000. For the $33 \mathrm{GHz}$ Tenerife data, we used the Metsahovi catalog 
(Metsahovi group, private communication) which regularly monitors sources above $1 \mathrm{Jy}$ at 22 and $37 \mathrm{GHz}$. This catalog is neither complete in flux nor time, although observations are available up to January 2000.

We have developed a software to produce time and frequency complete point source catalogs. The flux of the sources for each frequency was interpolated in time by fitting Fourier series to the data. If the number of independent observations per frequency was smaller than 10 , the sources were assumed not variable, and the data was fitted to a constant with time. The extrapolation of the source fluxes into the future time was performed assuming no variability since last observed data point. Spectral indices were calculated for each source from the available data. If only observations at a single frequency were available, we assumed a flat spectrum. We constructed four point source catalogs at 10, 13, 15, and $33 \mathrm{GHz}$ covering the time range from January 1984 to December 2000 with one Julian day sampling and a flux limit of about $0.5 \mathrm{Jy}$.

For each single day of observations with the Tenerife radiometers, we estimated the total contribution from point sources to the data. This contribution was calculated from the extrapolated catalog at 10,15, and $33 \mathrm{GHz}$ and the flux, $S$, was converted into antenna temperature, $T$, using [64]

$$
T=\frac{S \lambda^{2}}{2 k \Omega},
$$

where $\Omega$ is the beam solid angle, and $\lambda$ is the wavelength. The daily point source contributions were stacked in the same manner as the Tenerife data and subtracted from the Tenerife stacks. In the left column of Figure 5, we plot the Tenerife stacked data at $15 \mathrm{GHz}$ for the 10 declinations presented in this paper and overplot in green the contribution from point sources to the data. In the right column, we plot the stacks after subtraction of the point sources.

4.1. Unresolved Sources. A study of the contribution of unresolved point sources (i.e., weak point sources not detected individually) to $\mathrm{CMB}$ experiments has been produced by Franceschini et al. [65]. They used numerous surveys, including VLA and IRAS data, to put limits on the contribution to single beam CMB experiments by a random distribution of point sources. The contribution from unresolved point sources to the Tenerife data deduced from the previous analysis is presented in Table 4 . We consider sources with flux under $1 \mathrm{Jy}$ at $5 \mathrm{GHz}$ and a main beam of $5^{\circ}$.

\section{Assessment of the Galactic Contribution to the Tenerife Data}

To assess galactic contribution to the Tenerife data, we correlated observations with the galactic synchrotron, dust, and $H_{\alpha}$ emission templates described in the previous sections. We convolved the foreground maps with the Tenerife beam at each of the Tenerife frequencies before the correlation was performed. The Tenerife data used in the correlation are the final stacked data presented in Section 2. At $15 \mathrm{GHz}$ we used ten declination stacks in the range $25^{\circ}-47.5^{\circ}$; seven
TABLE 4: Contribution from unresolved point sources to the Tenerife experiment.

\begin{tabular}{lc}
\hline$v(\mathrm{GHz})$ & $\Delta T / T(\mu \mathrm{K})$ \\
\hline 10 & $\leq 7.5 \mu \mathrm{K}$ \\
15 & $\leq 4.0 \mu \mathrm{K}$ \\
33 & $\leq 0.6 \mu \mathrm{K}$ \\
\hline
\end{tabular}

declination stacks at $10 \mathrm{GHz}$ in the range $30^{\circ}-45^{\circ}$; and only two declination stacks at $33 \mathrm{GHz}$ covering declinations $40^{\circ}$ and $42.5^{\circ}$. The data were processed so that data at $|b| \leq$ 15 were excluded from the baseline fit and therefore were neither stacked or reconstructed. The discrete point sources were subtracted from the Tenerife stacks as discussed in the previous section.

5.1. The Method. To simultaneously correlate the Tenerife data to the three galactic templates, we use a method which was first applied to this problem by Górski et al. [66] to fit galactic and extragalactic templates in Fourier space to the COBE-DMR data. This method was applied to the Tenerife data first by de Oliveira-Costa et al. [29, 30] and then by Mukherjee et al. [39] to study the possible emission of spinning dust at the Tenerife frequencies.

Assuming that the microwave data is a superposition of $\mathrm{CMB}$, noise, and galactic components, we can write

$$
y=a X+x_{\mathrm{CMB}}+n,
$$

where $y$ is a Tenerife data vector of $N$ pixels; $X$ is an $N \times M$ element matrix containing $M=3$ foreground templates convolved with the Tenerife beam; $a$ is a vector of size $M$ that represents the levels at which these foreground templates are present in the Tenerife data-correlation coefficients for each foreground template; $n$ is the instrumental noise in the data; and $x_{\mathrm{CMB}}$ is the $\mathrm{CMB}$ component of the data. For this analysis, we assume the noise and CMB to be uncorrelated.

The minimum variance estimate of $a$ is given by

$$
\widehat{a}=\left[X^{T} C^{-1} X\right]^{-1} X^{T} C^{-1} y
$$

with errors given by $\sigma_{\widehat{a}_{i}}=\sum_{i i}^{1 / 2}$, where $\sum$ is given as

$$
\sum=\left\langle\widehat{a}^{2}\right\rangle-\langle\widehat{a}\rangle^{2}=\left[X^{T} C^{-1} X\right]^{-1} .
$$

In the before mentioned, $C$ is the total covariance matrix, the sum of the noise covariance matrix, and the CMB covariance matrix. The noise covariance matrix of the Tenerife data is taken to be diagonal-no correlation between different pixels. The CMB covariance matrix was obtained analytically following Zaldarriaga [67].

This correlation method produces minimum variance and unbiased estimates of $a$ if the following holds.

(i) The error in the Tenerife data is Gaussian and with zero mean. To a very good approximation the instrumental noise in the Tenerife data is uncorrelated. 

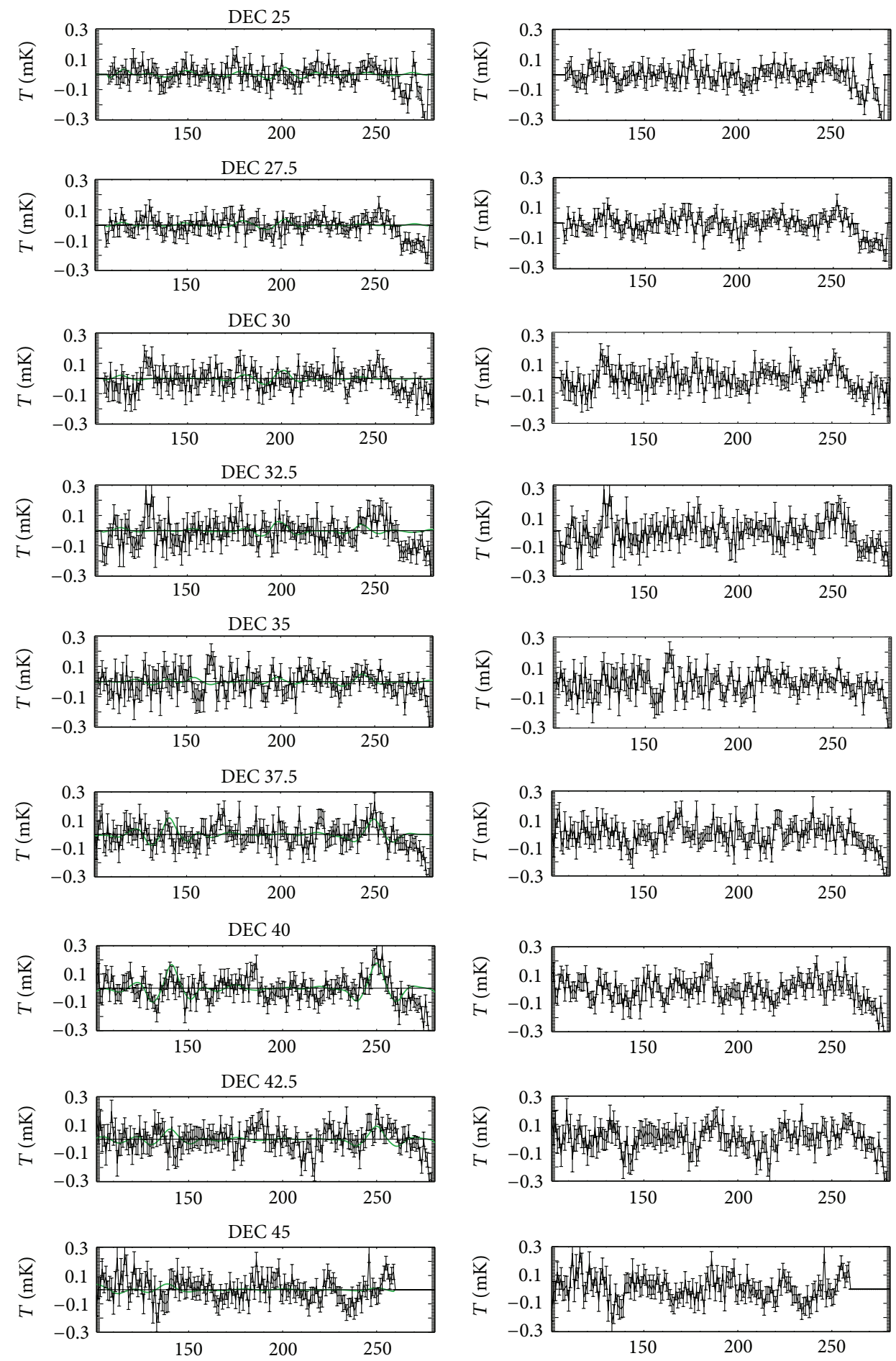

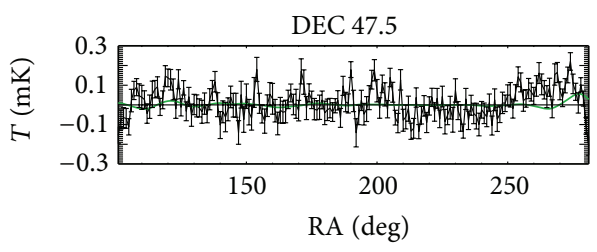

(a)

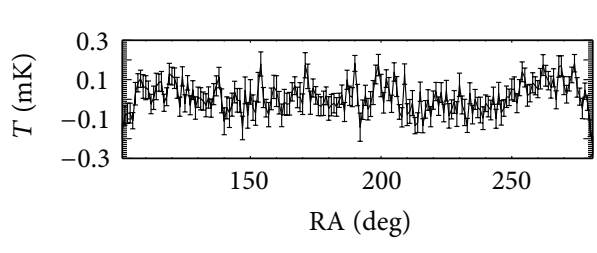

(b)

FIGURE 5: Extrapolated point source contribution to the $15 \mathrm{GHz}$ Tenerife data. (a): Observed scans; (b): point source-corrected scans. 
(ii) The templates perfectly trace the foreground emissions at microwave frequencies. There may be components of emission present in the Tenerife data, apart from those we have identified, for which there are not obvious counterparts at other frequencies. Moreover, the templates we use do not perfectly trace the microwave emission of the galactic foreground. The latter is specially important in the case of vibrational and rotational dust for which the template comes from data at much higher frequencies. For example, Draine and Lazarian [3] proposed that a $12 \mu \mathrm{m}$ map would be a much better tracer of rotational dust emission because small grains will also emit at this frequency. Also, the $H_{\alpha}$ emission is absorbed at low galactic latitudes by interstellar dust and therefore does not fully trace the free-free emission at those latitudes. Moreover, the $408 \mathrm{MHz}$ map has residual striping.

(iii) The templates are perfect-errors bars equal to zero. The error estimated for the templates are about 5\%$10 \%$ of the signal. They are not corrected for in this analysis because they are small compared to the errors in the Tenerife data.

(iv) The templates are not correlated. It there is a correlation between the different templates, the set of minimum variance correlation coefficients $\widehat{a}$ is degenerate and, therefore, we would not be able to discriminate among the different foreground components. At low galactic latitudes, the galactic plane emission dominates and Mukherjee et al. [39] have found that the foreground templates are correlated in this region.

(v) The correlation coefficient $a$ is the same throughout the area of sky for which the correlation is performed. If it is not, the error associated with $a$ is systematically underestimated. As a double check, we divided the sky observed into independent areas and performed the same correlation test in each, calculating a mean correlation coefficient and the dispersion of the individual values which in most cases was in good agreement with the error of $a$ calculated for the total area.

5.2. Cross-Correlation Results. The cross-correlation results are presented in Table 5. The correlation was performed for three different galactic cuts $b>20, b>30$, and $b>40$. $\sigma_{\text {gal }}$ represents the r.m.s. of the galactic maps after convolution with the Tenerife beam. $\widehat{a}$ and $\sigma_{\widehat{a}}$ are the correlation coefficient and the error associated with it. They have units of $\mu \mathrm{K} / \mathrm{K}, \mu \mathrm{K} /\left(\mathrm{MJy} \mathrm{sr}^{-1}\right)$ and $\mu \mathrm{K} / \mathrm{R}$ for the correlation with the $408 \mathrm{MHz}, 100 \mu \mathrm{m}$, and WHAM maps respectively. $\Delta T$ is the r.m.s. contribution from the galactic foregrounds to the Tenerife data, which was obtained as $\sigma_{\text {gal }} \times \widehat{a}$. This analysis is improved with respect to previous analyses by de OliveiraCosta et al. [29, 30] and Mukherjee et al. [39], First, because we present Tenerife data at 10 and $15 \mathrm{GHz}$ for a much larger area of the sky and with improved sensitivity; and second, because we also include in the cross-correlation a template
TABLE 5: Cross-correlation results. See text for details.

\begin{tabular}{|c|c|c|c|c|c|}
\hline \multicolumn{2}{|c|}{ Galactic cut $v(\mathrm{GHz})$} & \multicolumn{2}{|c|}{$\sigma_{\mathrm{gal}}$} & \multirow{2}{*}{$\frac{\widehat{a} \pm \sigma_{\widehat{a}}}{17 \pm 6}$} & \multirow{2}{*}{$\frac{\Delta T(\mu \mathrm{K})}{17.4 \pm 6.0}$} \\
\hline \multirow{9}{*}{$b>20^{\circ}$} & \multirow{3}{*}{$10 \mathrm{GHz}$} & $408 \mathrm{MHz}$ & 1.0217 & & \\
\hline & & $100 \mu \mathrm{m}$ & 0.3256 & $23 \pm 17$ & $7.5 \pm 5.5$ \\
\hline & & WHAM & 0.2513 & $42 \pm 20$ & $10.5 \pm 5.5$ \\
\hline & \multirow{3}{*}{$15 \mathrm{GHz}$} & $408 \mathrm{MHz}$ & 1.0244 & $9 \pm 3$ & $9.2 \pm 3.0$ \\
\hline & & $100 \mu \mathrm{m}$ & 0.3308 & $66 \pm 8$ & $21.8 \pm 2.6$ \\
\hline & & WHAM & 0.2758 & $-10 \pm 10$ & $-2.7 \pm 2.7$ \\
\hline & \multirow{3}{*}{$33 \mathrm{GHz}$} & $408 \mathrm{MHz}$ & 0.8904 & $4 \pm 9$ & $3.6 \pm 8.0$ \\
\hline & & $100 \mu \mathrm{m}$ & 0.3089 & $86 \pm 27$ & $26.0 \pm 8.0$ \\
\hline & & WHAM & 0.2768 & $21 \pm 51$ & $5.8 \pm 10.0$ \\
\hline \multirow{9}{*}{$b>30^{\circ}$} & \multirow{3}{*}{$10 \mathrm{GHz}$} & $408 \mathrm{MHz}$ & 0.8722 & $15 \pm 7$ & $13.0 \pm 6.0$ \\
\hline & & $100 \mu \mathrm{m}$ & 0.2028 & $72 \pm 30$ & $14.6 \pm 6.0$ \\
\hline & & WHAM & 0.1435 & $83 \pm 42$ & $11.9 \pm 6.0$ \\
\hline & \multirow{3}{*}{$15 \mathrm{GHz}$} & $408 \mathrm{MHz}$ & 0.8544 & $13 \pm 4$ & $11.1 \pm 3.4$ \\
\hline & & $100 \mu \mathrm{m}$ & 0.2036 & $-1 \pm 14$ & $-0.2 \pm 2.8$ \\
\hline & & WHAM & 0.1504 & $30 \pm 20$ & $4.5 \pm 3.0$ \\
\hline & \multirow{3}{*}{$33 \mathrm{GHz}$} & $408 \mathrm{MHz}$ & 0.7749 & $7 \pm 10$ & $5.4 \pm 7.7$ \\
\hline & & $100 \mu \mathrm{m}$ & 0.2143 & $90 \pm 45$ & $19.2 \pm 9.6$ \\
\hline & & WHAM & 0.1473 & $17 \pm 63$ & $2.5 \pm 9.3$ \\
\hline \multirow{9}{*}{$b>40^{\circ}$} & \multirow{3}{*}{$10 \mathrm{GHz}$} & $408 \mathrm{MHz}$ & 0.7676 & $11 \pm 9.5$ & $8.4 \pm 8.0$ \\
\hline & & $100 \mu \mathrm{m}$ & 0.1898 & $110 \pm 45$ & $20.9 \pm 8.5$ \\
\hline & & WHAM & 0.1292 & $77 \pm 52$ & $9.9 \pm 6.7$ \\
\hline & \multirow{3}{*}{$15 \mathrm{GHz}$} & $408 \mathrm{MHz}$ & 0.7412 & $15 \pm 5$ & $11.1 \pm 3.7$ \\
\hline & & $100 \mu \mathrm{m}$ & 0.1782 & $-11 \pm 21$ & $-1.9 \pm 3.7$ \\
\hline & & WHAM & 0.1354 & $51 \pm 25$ & $6.9 \pm 3.4$ \\
\hline & \multirow{3}{*}{$33 \mathrm{GHz}$} & $408 \mathrm{MHz}$ & 0.6630 & $4 \pm 15$ & $2.6 \pm 9.9$ \\
\hline & & $100 \mu \mathrm{m}$ & 0.2138 & $66 \pm 55$ & $14.8 \pm 11.8$ \\
\hline & & WHAM & 0.1499 & $47 \pm 69$ & $7.0 \pm 10.3$ \\
\hline
\end{tabular}

for the free-free emission. Moreover, data at $33 \mathrm{GHz}$ have also been included in the analysis although the area of the sky observed is significantly smaller than at 10 and $15 \mathrm{GHz}$ and consequently the error bars in the estimated cross-correlation coefficients are much larger. We have pictorially summarised the cross-correlation results in Figure 6. In the top row of the figure, we plot the r.m.s. contribution from synchrotron to the Tenerife data at the three galactic cuts analysed. In black, we overplot the expected synchrotron contribution for a spectral index of -3.0 and derived from the r.m.s. level in the $408 \mathrm{MHz}$ map. The contributions are systematically lower than expected at $10 \mathrm{GHz}$, and the spectral index is much flatter such that, for example, the contribution at $15 \mathrm{GHz}$ is consistent with that at $10 \mathrm{GHz}$ for $b>40^{\circ}$. We have derived from the Tenerife data spectral indexes of $-1.77_{-2.0}^{+1.0},-0.40_{-1.9}^{+1.0}$ and $-0.40_{-1.6}^{+2.0}$ at $b>20^{\circ}, b>30^{\circ}$ and $b>40^{\circ}$ respectively, and the r.m.s contributions based on these spectral indices is overplotted in red.

In the middle row, we plot the r.m.s. contribution from free-free emission to the Tenerife data. In black, we overplot the expected free-free emission at microwave frequencies derived from (2) which are actually in very good agreement 
TABLE 6: Cross-correlation results of CMB data with dust. Available cross-correlation coefficients of CMB data sets with dust templates at $100 \mu \mathrm{m}$.

\begin{tabular}{|c|c|c|c|c|}
\hline Experiment & Frequency $(\mathrm{GHz})$ & $|b|>$ (degrees) & $\widehat{a} \pm \sigma_{\widehat{a}} \mu \mathrm{K} /\left(\mathrm{MJy} \cdot \mathrm{sr}^{-1}\right)$ & References \\
\hline COBE DMR & 31.5 & 20 & $18.0 \pm 2.5$ & Kogut et al. [37] \\
\hline COBE DMR & 31.5 & 30 & $14.5 \pm 6.0$ & Kogut et al. [37] \\
\hline COBE DMR & 53.0 & 20 & $6.8 \pm 1.4$ & Kogut et al. [37] \\
\hline COBE DMR & 53.0 & 30 & $6.4 \pm 3.4$ & Kogut et al. [37] \\
\hline COBE DMR & 90.0 & 20 & $2.7 \pm 1.6$ & Kogut et al. [37] \\
\hline COBE DMR & 90.0 & 30 & $4.6 \pm 3.9$ & Kogut et al. [37] \\
\hline Saskatoon (Ka band) & 30.0 & NCP & $15.0 \pm 8.1$ & De Oliveira-Costa et al. $[25,26]$ \\
\hline Saskatoon (Q band) & 40.0 & $\mathrm{NCP}$ & $11.8 \pm 10$ & De Oliveira-Costa et al. $[25,26]$ \\
\hline $19 \mathrm{GHz}$ survey & 19.0 & 20 & $38.5 \pm 3.5$ & De Oliveira-Costa et al. $[27,28]$ \\
\hline $19 \mathrm{GHz}$ survey & 19.0 & 30 & $47.1 \pm 9.0$ & De Oliveira-Costa et al. $[27,28]$ \\
\hline OVRO & 14.5 & $\mathrm{NCP}$ & 209 & Leitch et al. $[1,2]$ \\
\hline OVRO & 32.0 & $\mathrm{NCP}$ & 36 & Leitch et al. $[1,2]$ \\
\hline PYTHON V & 40.3 & & $-3.0 \pm 18.0$ & Coble et al. [38] \\
\hline Tenerife & 10.0 & 20 & $49.8 \pm 11.0$ & De Oliveira-Costa et al. $[29,30]$ \\
\hline Tenerife & 10.0 & 30 & $-8.3 \pm 31.0$ & De Oliveira-Costa et al. $[29,30]$ \\
\hline Tenerife & 10.0 & 40 & $84.0 \pm 54.0$ & De Oliveira-Costa et al. $[29,30]$ \\
\hline Tenerife & 15.0 & 20 & $71.8 \pm 4.5$ & De Oliveira-Costa et al. $[29,30]$ \\
\hline Tenerife & 15.0 & 30 & $94.9 \pm 15.0$ & De Oliveira-Costa et al. $[29,30]$ \\
\hline Tenerife & 15.0 & 40 & $72.0 \pm 33.0$ & De Oliveira-Costa et al. $[29,30]$ \\
\hline Tenerife & 10.0 & 20 & $71.0 \pm 18.0$ & Mukherjee et al. [39] \\
\hline Tenerife & 10.0 & 30 & $-7.0 \pm 32.0$ & Mukherjee et al. [39] \\
\hline Tenerife & 10.0 & 40 & $28.0 \pm 39.0$ & Mukherjee et al. [39] \\
\hline Tenerife & 15.0 & 20 & $91.0 \pm 11.0$ & Mukherjee et al. [39] \\
\hline Tenerife & 15.0 & 30 & $29.0 \pm 20.0$ & Mukherjee et al. [39] \\
\hline Tenerife & 15.0 & 40 & $3.0 \pm 26.0$ & Mukherjee et al. [39] \\
\hline South Pole 94 & 30.0 & 40 & $20.0 \pm 36.0$ & Hamilton and Ganga [40] \\
\hline South Pole 94 & 40.0 & 40 & $68.1 \pm 42.4$ & Hamilton and Ganga [40] \\
\hline
\end{tabular}

with the observations. In red, we plot the free-free contribution for spectral indexes of $-0.5,-2.20$, and -1.0 at $b>20^{\circ}$, $b>30^{\circ}$, and $b>40^{\circ}$, respectively.

In the bottom row of Figure 6, we plot the r.m.s. contribution from dust emission to the Tenerife data which is significantly larger (few orders of magnitude) than expected from vibrational dust. In solid black, we overplot an r.m.s brightness temperature expected from the CNM model of rotational dust proposed by Draine and Lazarian [3]. The models were rescaled to fit the data. The intensity spectrum for this model peaks at $50 \mathrm{GHz}$ which corresponds to a peak in brightness temperature around $20 \mathrm{GHz}$ of about $300 \mu \mathrm{K}$. From the best fit model to the data, we have estimated fluctuations in the temperature of the rotational dust of $\sim 8 \%$ at the angular scales of the Tenerife experiment.

The r.m.s. contributions from dust to the $15 \mathrm{GHz}$ data at $b>30^{\circ}$ and $b>40^{\circ}$, as well as the contribution from freefree at $b>20^{\circ}$ seem to be underestimated. This could be caused by correlations between the synchrotron, dust, and free-free templates which would reduce the validity of the minimum variance solution and could bias the estimates of the correlation. In Figure 7, we plot the following correlations, from top to bottom synchrotron versus dust; free-free versus dust, and free-free versus synchrotron for $b>20^{\circ}$, $b>30^{\circ}$, and $b>40^{\circ}$. We observe no correlation between freefree and synchrotron, moderate correlation between free-free and dust and a quite strong correlation between synchrotron and dust at low galactic latitudes weakening down at high latitudes. Note that at $b>20^{\circ}$, the correlation plots show negative versus negative points which do not follow the correlation pattern of the main body of points. The former points correspond to the lowest galactic latitude data which run into a negative beam of the triple beam pattern for the galactic plane crossing. The observed correlations between templates can not justify the observed lack of dust correlation at $15 \mathrm{GHz}$ data at $b>30^{\circ}$ and $b>40^{\circ}$. In the following, we concentrate on the $b>20^{\circ}$ region for which we detect significant dust correlated signal at all Tenerife frequencies.

\section{Dust-Correlated Emission}

Hinshaw et al. [37] cross-correlated the COBE differential microwave radiometer (DMR) maps with DIRBE far infrared maps and discovered that statistically significant correlation did exist at each DMR frequency, which was inconsistent with vibrational dust alone. This extra correlation was explained 

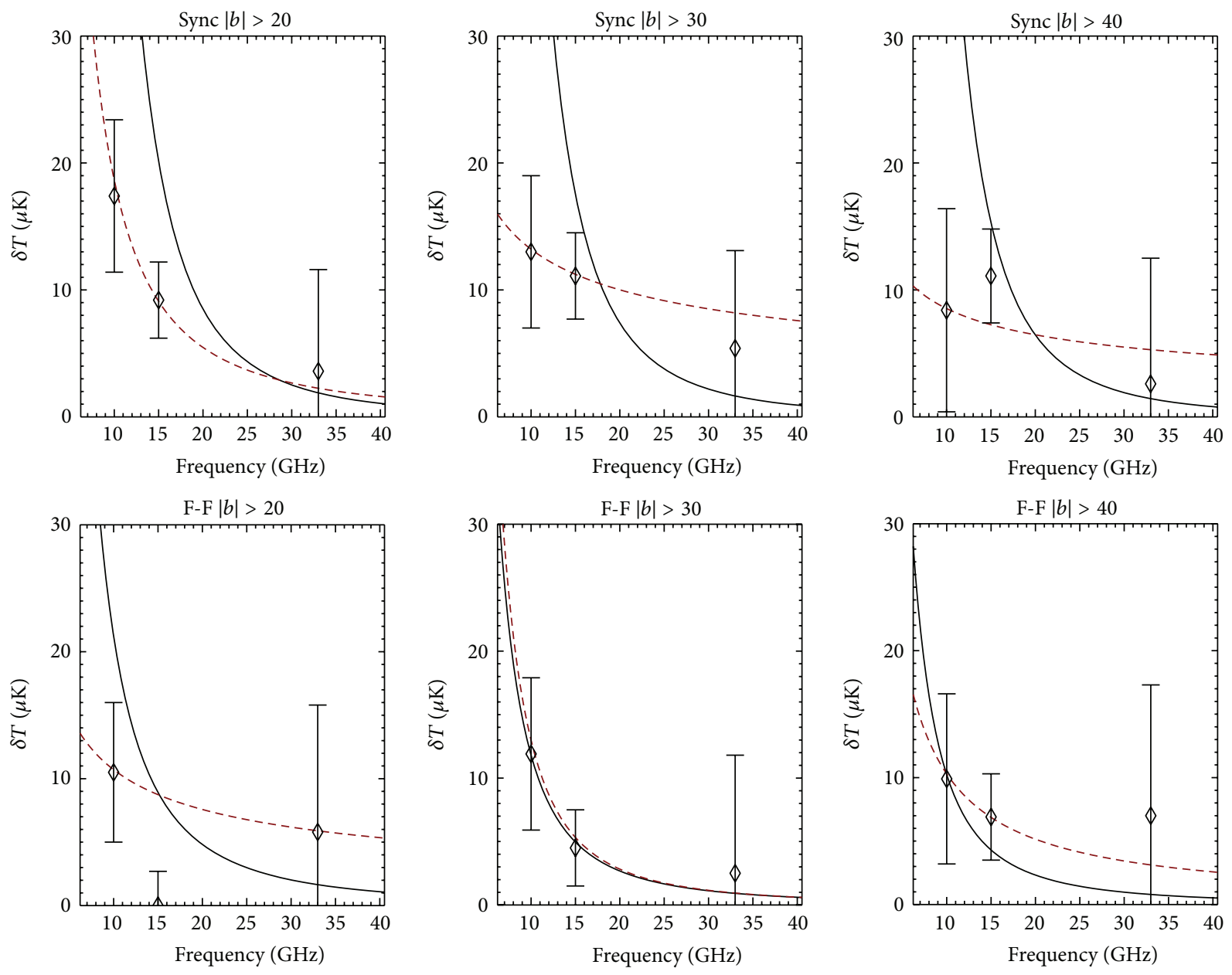

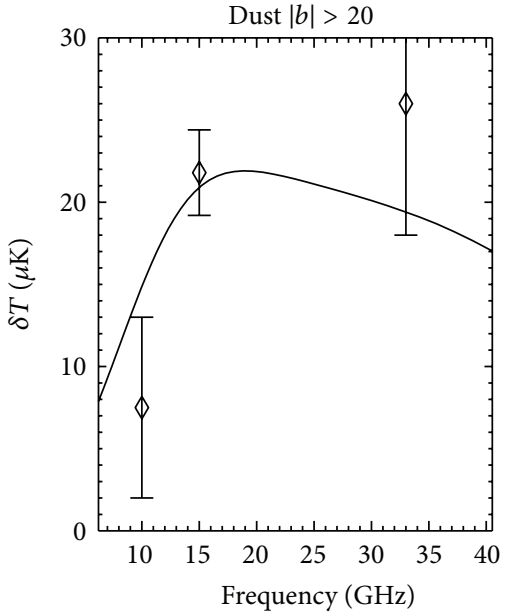

(a)

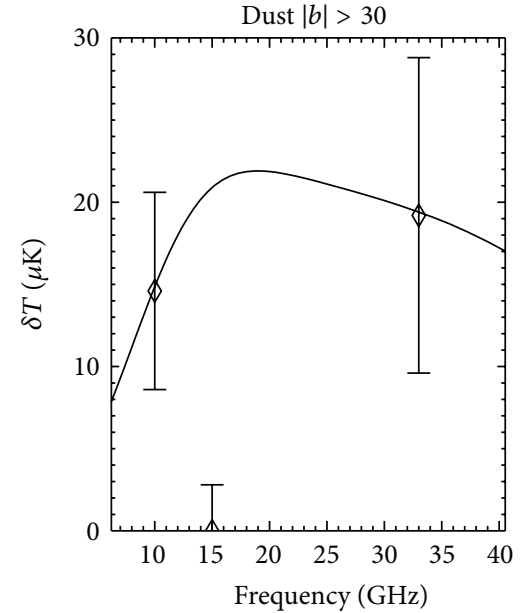

(b)

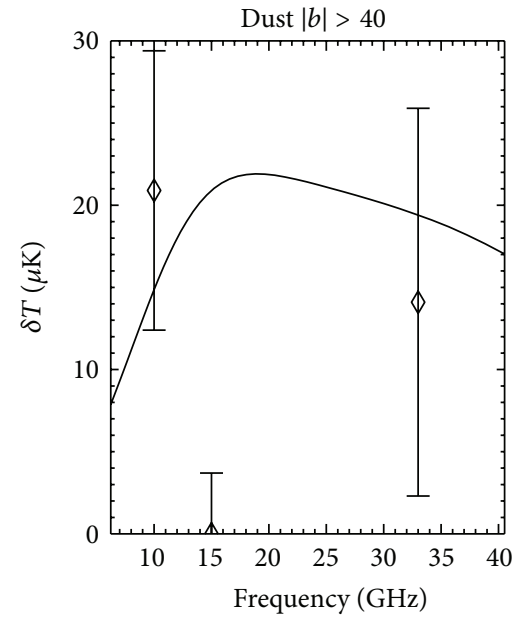

(c)

FIGURE 6: Spectra of foregrounds at the Tenerife frequencies. We represent the cross-correlation coefficients as a function of frequency for $b>20, b>30$, and $b>40$ ((a), (b), and (c), resp.). From top to bottom, we plot the r.m.s. contribution from synchrotron, free-free, and dust to theTenerife data. In solid black and red, we show standard and best-fit models of the electromagnetic spectrum for each galactic emission. The models for dust are rescaled to fit the data. Details on the best-fit models can be found in the text. 

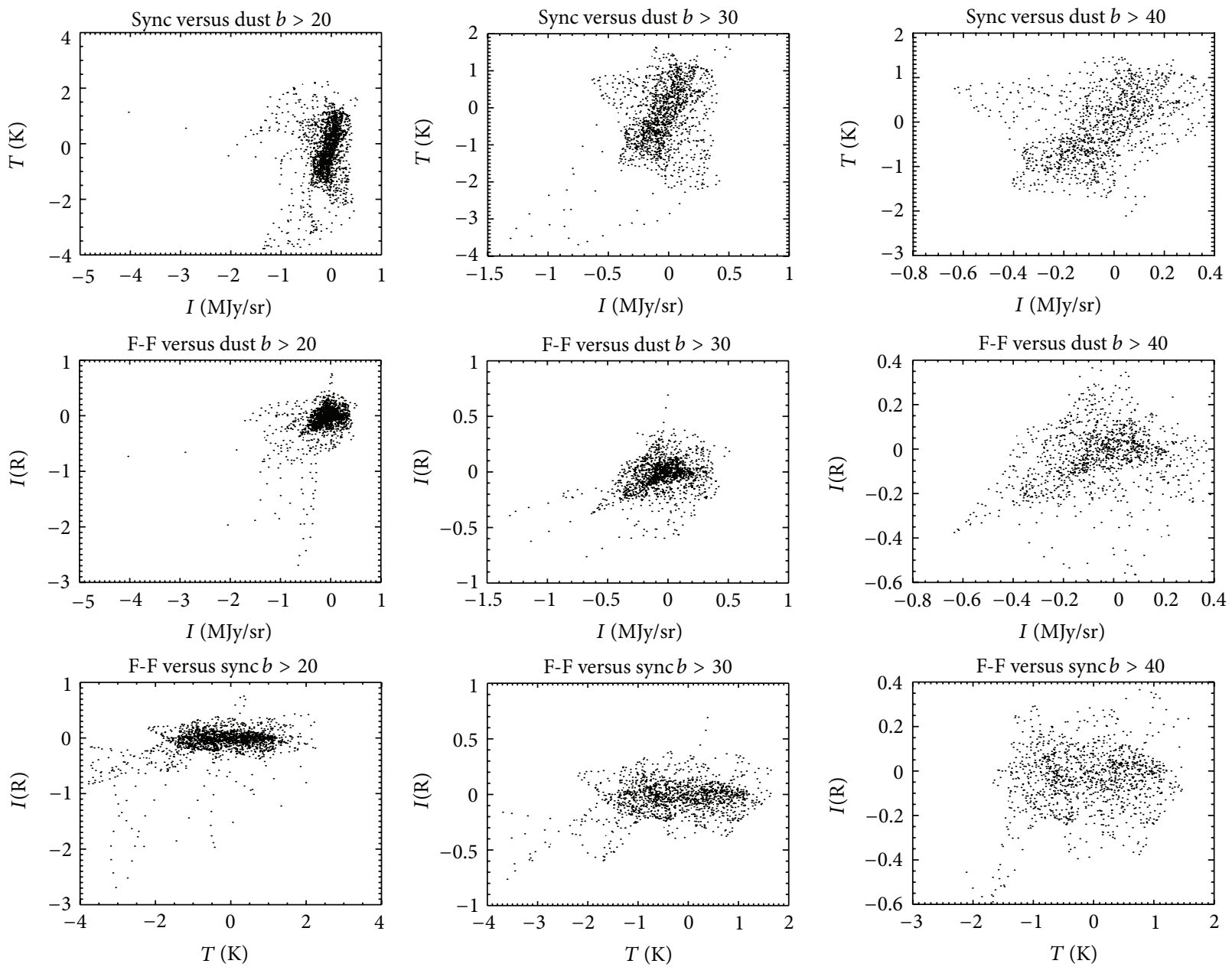

(a)

(b)

(c)

FIGURE 7: Correlation between the foreground templates. From top to bottom, we plot synchrotron versus dust, free-free versus dust and free-free versus synchrotron. The correlation is presented for $b>20, b>30$, and $b>40$ ((a), (b), and (c), resp.).

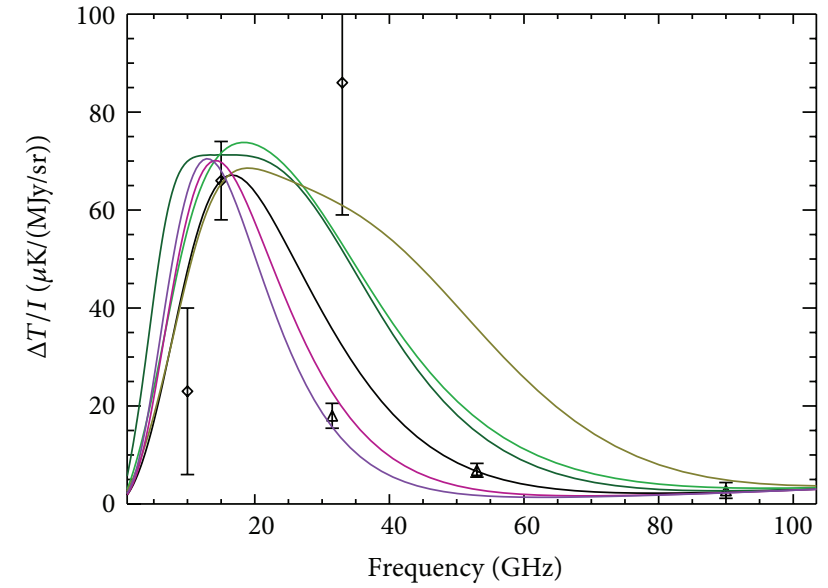

FIGURE 8: Cross-correlation coefficients for the dust-correlated emission. We plot the Tenerife data as diamonds and the COBE DMR data as triangles. The color solid curves over plotted correspond to joint vibrational and rotational dust models as described in the text. at the time as free-free emission. Following this publication, other authors have cross-correlated CMB data sets with dust templates and found an excess of correlation which has been interpreted as free-free emission, flat-spectrum synchrotron, or emission from spinning dust. In Table 6, we present an up-to-date list of $\mathrm{CMB}$ data for which the correlation with dust has been performed. In all cases, a multitemplate method has been used to perform the correlation but no free-free template has been used. We have also added in this table previous analyses of the Tenerife data by de Oliveira-Costa et al. [29, 30] and Mukherjee et al. [39] which did not include the $33 \mathrm{GHz}$ data and covered a much smaller area of the sky at 10 and $15 \mathrm{GHz}$.

The correlation coefficients we deduced are significantly smaller at $|b|>20$ than those calculated by de Oliveira-Costa et al. [29, 30] and Mukherjee et al. [39]. This is probably due to $50 \%$ larger area (localized signals are diluted) and a more careful subtraction of baselines at low galactic latitudes in our data. The inclusion of an extra free-free template seems to play a minor role on this at $10 \mathrm{GHz}$ and none at $15 \mathrm{GHz}$. 
The cross-correlation results for the Tenerife experiment presented in this paper confirm the existence of extra dustcorrelated emission at microwave frequencies and suggest that it is not due to free-free emission. The moderate correlation found between the dust and free-free templates could confuse the results obtained but clearly cannot account for all the observed dust-correlated component. A more detailed study is needed to take into account correlations between templates in the calculation of the correlation coefficients.

In Figure 8, we plot the dust cross-correlation coefficients $(|b|>20)$ for the Tenerife (diamonds) and COBE-DMR (triangles) data. The rest of the experiments presented in Table 6 were not included in this plot because they observe at different angular scales and different areas of the sky (see table for details) and therefore no direct comparison with the Tenerife and COBE data is possible. The Tenerife data suggest a peak in the spectrum at about $30 \mathrm{GHz}$ although this is mainly based on the data at $33 \mathrm{GHz}$ which is significantly much noisier and cover a much smaller part of the sky. However, DMR data combined with the 10 and $15 \mathrm{GHz}$ Tenerife data points prefer a peak in the range $15-20 \mathrm{GHz}$. In color, we overplot the six spinning dust models proposed by Draine and Lazarian [3] combined with the standard vibrational dust model described in the previous sections, for which the amplitude has been taken as a free parameter and fitted to the COBE and Tenerife 10 and $15 \mathrm{GHz}$ data. We observe that the shape of the spectrum given by the data is similar to some of the predicted spectra although the models do not indicate a sharp rise in the range $10-15 \mathrm{GHz}$.

\section{Conclusions}

We have presented in this paper a reanalysis of the Tenerife data including previously published data and new data from January 1998 to December 2000. This analysis leads to evidences for an excess of dust-correlated emission at microwave frequencies in the range $10-33 \mathrm{GHz}$ and at large angular scales, from 5 to 15 degrees. This correlation can not all be associated with free-free emission. A combination of the Tenerife and COBE data suggests spinning dust emission could account for the extra correlation. However, the scatter observed in the data and the discrepancy in the spectrum shape indicate other components may also be responsible for the extra correlation. Furthermore, the analysis does not take into account correlations between the galactic emission templates and this can bias the estimate of the correlation coefficients. To correct this, a more detailed analysis, which will account for the cross-correlation between templates, is needed.

\section{Acknowledgment}

The authors would like to thank the editors of this paper.

\section{References}

[1] E. M. Leitch, A. C. S. Readhead, T. J. Pearson, and S. T. Myers, "An anomalous component of galactic emission," The Astrophysical Journal, vol. 486, no. 1, pp. 23-26, 1997.
[2] E. M. Leitch, A. C. S. Readhead, T. J. Pearson, and S. T. Myers, "An anomalous component of galactic emission," Astrophysical Journal Letters, vol. 486, no. 1, pp. L23-L26, 1997.

[3] B. T. Draine and A. Lazarian, "Diffuse Galactic emission from spinning dust grains," The Astrophysical Journal Letters, vol. 494, no. 1, pp. L19-L22, 1998.

[4] B. T. Draine and A. Lazarian, "Electric dipole radiation from spinning dust grains," The Astrophysical Journal, vol. 508, no. 1, pp. 157-179, 1998.

[5] Douglas P. Finkbeiner, "A full-sky $\mathrm{H} \alpha$ template for microwave foreground prediction," The Astrophysical Journal Supplement, vol. 146, no. 2, pp. 407-415, 2003.

[6] A. De Oliveira-Costa, M. Tegmark, R. D. Davies et al., "The quest for microwave foreground X," Astrophysical Journal Letters, vol. 606, no. 2, pp. L89-L92, 2004.

[7] R. A. Watson, R. Rebolo, J. A. Rubiño-Martín et al. et al., "Detection of anomalous microwave emission in the perseus molecular cloud with the COSMOSOMAS experiment," The Astrophysical Journal Letters, vol. 624, no. 2, pp. L89-L92, 2005.

[8] S. Iglesias-Groth, "Electric dipole emission by fulleranes and galactic anomalous microwave emission," The Astrophysical Journal Letters, vol. 632, no. 1, pp. L25-L28, 2005.

[9] S. Casassus, G. F. Cabrera, F. Förster, T. J. Pearson, A. C. S. Readhead, and C. Dickinson, "Morphological analysis of the centimeter-wave continuum in the dark cloud LDN 1622," The Astrophysical Journal, vol. 639, no. 2, pp. 951-964, 2006.

[10] S. Casassus, C. Dickinson, K. Cleary et al. et al., "Centimetrewave continuum radiation from the $\rho$ Ophiuchi molecular cloud," Monthly Notices of the Royal Astronomical Society, vol. 391, no. 3, pp. 1075-1090, 2008.

[11] C. Dickinson, R. D. Davies, J. R. Allison et al. et al., "Anomalous microwave emission from the HII region RCW175," The Astrophysical Journal, vol. 690, no. 2, pp. 1585-1589, 2009.

[12] C. T. Tibbs, R. A. Watson, C. Dickinson et al., "Very small array observations of the anomalous microwave emission in the Perseus region," Monthly Notices of the Royal Astronomical Society, vol. 402, no. 3, pp. 1969-1979, 2010.

[13] Y. Ali-Haïmoud, C. M. Hirata, and C. Dickinson, "A refined model for spinning dust radiation," Monthly Notices of the Royal Astronomical Society, vol. 395, no. 2, pp. 1055-1078, 2009.

[14] T. Hoang, B. T. Draine, and A. Lazarian, "Improving the model of emission from spinning dust: effects of grain wobbling and transient spin-up," Astrophysical Journal Letters, vol. 715, no. 2, pp. 1462-1485, 2010.

[15] T. Hoang, A. Lazarian, and B. T. Draine, "Spinning dust emission: effects of irregular grain shape, transient heating and comparison with WMAP results," The Astrophysical Journal, vol. 741, no. 2, p. 87, 2011.

[16] B. T. Draine and A. Lazarian, "Magnetic dipole microwave emission from dust grains," Astrophysical Journal Letters, vol. 512, no. 2, pp. 740-754, 1999.

[17] E. S. Battistelli, R. Rebolo, J. A. Rubiño-Martín et al., "Polarization observations of the anomalous microwave emission in the perseus molecular complex with the COSMOSOMAS experiment," The Astrophysical Journal Letters, vol. 645, no. 2, pp. L141-L144, 2006.

[18] A. Kogut, J. Dunkley, C. L. Bennett et al., “Three-year wilkinson microwave anisotropy probe (WMAP) observations: foreground polarization," The Astrophysical Journal, vol. 665, no. 1, pp. 335-362, 2007. 
[19] B. S. Mason, T. Robishaw, C. Heiles, D. Finkbeiner, and C. Dickinson, "A limit on the polarized anomalous microwave emission of lynds 1622," Astrophysical Journal Letters, vol. 697, no. 2, pp. 1187-1193, 2009.

[20] C. H. López-Caraballo, J. A. Rubiño-Martín, R. Rebolo, and R. Génova-Santos, "Constraints on the polarization of the anomalous microwave emission in the perseus molecular complex from seven-year WMAP data," The Astrophysical Journal, vol. 729, no. 1, p. 25, 2011.

[21] B. T. Draine and B. Hensley, "Magnetic nanoparticles in the interstellar medium: emission spectrum and polarization," The Astrophysical Journal, vol. 765, no. 2, p. 159, 2013.

[22] F. X. Desert, F. Boulanger, and J. L. Puget, Eds., "Interstellar dust models for extinction and emission," Astronomy \& Astrophysics, vol. 237, no. 1, pp. 2015-236, 1990.

[23] A. Kogut, A. J. Banday, C. L. Bennett, K. M. Gorski, G. Hinshaw, and W. T. Reach, "High-Latitude Galactic Emission in the COBE Differential Microwave Radiometer 2 Year Sky Maps," The Astrophysical Journal, vol. 460, 1 pages, 1996.

[24] A. Kogut, A. J. Banday, C. L. Bennett et al., "Microwave emission at high galactic latitudes in the four-year DMR sky maps," The Astrophysical Journal Letters, vol. 464, no. 1, pp. L5-L19, 1996.

[25] M. S. Kowitt, E. S. Cheng, D. A. Cottingham et al., "A detection of bright features in the microwave background," The Astrophysical Journal, vol. 482, no. 1, pp. 17-21, 1997.

[26] A. De Oliveira-Costa, A. Kogut, M. J. Deviln, C. Barth Netterfield, L. A. Page, and E. J. Wollack, "Galactic microwave emission at degree angular scales," Astrophysical Journal Letters, vol. 482, no. 1, pp. L17-L20, 1997.

[27] A. de Olivera-Costa, M. Tegmark, L. A. Page, and S. P. Boughn, "Galactic emission at $19 \mathrm{GHz}$," Astrophysical Journal, vol. 509, no. 1, pp. 9-12, 1998.

[28] A. de Olivera-Costa, M. Tegmark, L. A. Page, and S. P. Boughn, "Galactic emission at $19 \mathrm{GHz}$," Astrophysical Journal Letters, vol. 509, no. 1, pp. L9-L12, 1998.

[29] A. de Oliveira-Costa, M. Tegmark, C. M. Gutierrez et al., "Cross-correlation of Tenerife data with Galactic templatesevidence for spinning dust?” The Astrophysical Journal Letters, vol. 527, no. 1, pp. L9-L12, 1999.

[30] A. de Oliveira-Costa, M. Tegmark, C. M. Gutierrez et al., "Cross-correlation of Tenerife data with Galactic templatesevidence for spinning dust?” The Astrophysical Journal, vol. 527, no. 1, pp. 9-12, 1999.

[31] R. Genova-Santos, R. Rebolo, J. A. Rubino-Martin, C. H. LopezCaraballo, and S. R. Hildebrandt, "Detection of anomalous microwave emission in the pleiades reflection nebula with Wilkinson Microwave Anisotropy Probe and the COSMOSOMAS experiment," Astrophysical Journal, vol. 743, no. 1, p. 67, 2011.

[32] A. M. M. Scaife, N. Hurley-Walker, D. A. Green et al., "An excess of emission in the dark cloud LDN1111 with the Arcminute Microkelvin Imager," Monthly Notices of the Royal Astronomical Society, vol. 394, no. 1, pp. L46-L50, 2009.

[33] A. M. I. Consortium, A. M. M. Scaife, N. Hurley-Walker et al. et al., "AMI observations of Lynds dark nebulae: further evidence for anomalous cm-wave emission," Monthly Notices of the Royal Astronomical Society, vol. 400, no. 3, pp. 1394-1412, 2009.

[34] P. Castellanos, S. Casassus, C. Dickinson et al. et al., "Dustcorrelated centimetre-wave radiation from the M78 reflection nebula," Monthly Notices of the Royal Astronomical Society, vol. 411, no. 2, pp. 1137-1150, 2011.
[35] Planck Collaboration, "Planck early results. XX. New light on anomalous microwave emission from spinning dust grains," Astronomy \& Astrophysics, vol. 536, article A20, 17 pages, 2011.

[36] C. Bot, N. Ysard, D. Paradis et al., "Submillimeter to centimeter excess emission from the Magellanic Clouds. II. On the nature of the excess," Astronomy \& Astrophysics, vol. 523, article A20, pp. 1-7, 2010.

[37] G. Hinshaw, A. J. Banday, C. L. Bennett et al., "Band power spectra in the COBE 1 DMR four-year anisotropy maps," Astrophysical Journal Letters, vol. 464, no. 1, pp. L17-L20, 1996.

[38] K. Coble, M. Dragovan, J. Kovac et al. et al., "Anisotropy in the cosmic microwave background at degree angular scales: python V results," The Astrophysical Journal Letters, vol. 519, no. 1, pp. L5-L8, 1999.

[39] P. Mukherjee, A. W. Jones, R. Kneissl, and A. N. Lasenby, “On dust-correlated Galactic emission in the Tenerife data," Monthly Notices of the Royal Astronomical Society, vol. 320, no. 2, pp. 224234, 2001.

[40] J. C. Hamilton and K. M. Ganga, "Correlation of the South Pole 94 data with $100 \mu \mathrm{m}$ and $408 \mathrm{MHz}$ maps," Astronomy \& Astrophysics, vol. 368, no. 3, pp. 760-765, 2001.

[41] C. L. Bennett, M. Halpern, G. Hinshaw et al. et al., "First-year wilkinson microwave anisotropy probe (WMAP) observations: preliminary maps and basic results," The Astrophysical Journal Supplement Series, vol. 148, no. 1, pp. 1-27, 2003.

[42] S. Fernández-Cerezo, C. M. Gutierrez, R. Rebolo et al., "Observations of the cosmic microwave background and galactic foregrounds at $12-17 \mathrm{GHz}$ with the COSMOSOMAS experiment," Monthly Notices of the Royal Astronomical Society, vol. 370, no. 1, pp. 15-24, 2006.

[43] S. R. Hildebrandt, R. Rebolo, J. A. Rubiño-Martín et al., "COSMOSOMAS observations of the cosmic microwave background and Galactic foregrounds at $11 \mathrm{GHz}$ : evidence for anomalous microwave emission at high Galactic latitude," Monthly Notices of the Royal Astronomical Society, vol. 382, no. 2, pp. 594-608, 2007.

[44] N. Ysard, M. A. Miville-Deschênes, and L. Verstraete, "Probing the origin of the microwave anomalous foreground," Astronomy \& Astrophysics, vol. 509, article L1, 2010.

[45] R. D. Davies, C. Dickinson, A. J. Banday, T. R. Jaffe, K. M. Górski, and R. J. Davis, "A determination of the spectra of Galactic components observed by the Wilkinson Microwave Anisotropy Probe," Monthly Notices of the Royal Astronomical Society, vol. 370, no. 3, pp. 1125-1139, 2006.

[46] A. Kogut, D. J. Fixsen, S. M. Levin et al., "Arcade 2 observations of galactic radio emission," The Astrophysical Journal, vol. 734, no. 1, p. 4, 2011.

[47] Carlos M. Gutierrez, R. Rebolo, Robert A. Watson, Rod D. Davies, Aled W. Jones, and Anthony N. Lasenby, "The Tenerife cosmic microwave background maps: observations and first analysis," The Astrophysical Journal, vol. 529, no. 1, pp. 47-55, 2000.

[48] S. Hancock, R. D. Davies, A. N. Lasenby et al., "Direct observation of structure in the cosmic microwave background," Nature, vol. 367, no. 6461, pp. 333-338, 1994.

[49] G. Ribicki and A. Lightman, Radiative Processes in Astrophysics, Wiley, New York, NY, USA, 1979.

[50] M. Longair, High Energy Astrophysics, Cambridge University Press, 1994.

[51] G. De Zotti, L. Toffolatti, F. Argüeso et al., "The Planck Surveyor mission: astrophysical prospects," AIP Conference Proceedings, vol. 476, pp. 204-223, 1999. 
[52] K. Lawson, C. Mayer, J. Osborn, and M. Parkinson, "Variations in the Spectral Index of the Galactic Radio Continuum Emission in the Northern Hemisphere," Monthly Notices of the Royal Astronomical Society, vol. 286, p. 307, 1987.

[53] C. G. T. Haslam, U. Klein, C. J. Salter et al., "A $408 \mathrm{MHz}$ all-sky continuum survey. I: observations at southern declinations and for the North Polar region," Astronomy \& Astrophysics, vol. 100, no. 2, pp. 209-219, 1981.

[54] P. Reich and W. Reich, "A map of spectral indices of the Galactic radio continuum emission between $408 \mathrm{MHz}$ and $1420 \mathrm{MHz}$ for the entire northern sky," Astronomy \& Astrophysics, vol. 74, p. 4, 1988.

[55] R. D. Davies, R. A. Watson, and C. M. Gutierrez, "Galactic synchrotron emission at high frequencies," Monthly Notices of the Royal Astronomical Society, vol. 278, no. 4, pp. 925-939, 1996.

[56] F. R. Bouchet and R. Gispert, "Foregrounds and CMB experiments: I. Semi-analytical estimates of contamination," New Astronomy, vol. 4, no. 6, pp. 443-479, 1999.

[57] A. Lasenby, "Radio frequency foreground emission and the separation of microwave anisotropies from foreground contamination," in Proceedings of the 16th Moriond Astrophysics Meeting: Microwave Background Anisotropies, vol. 453, 1996.

[58] D. Valls-Gabaud, "Cosmological applications of $\mathrm{H} \alpha$ surveys," Publications of the Astronomical Society of Australia, vol. 15, no. 1, pp. 111-117, 1998.

[59] L. M. Haffner, R. J. Reynolds, and S. L. Tufte, "WHAM observations of H-alpha, [S II], and [N II] toward the Orion and perseus arms: probing the physical conditions of the warm ionized medium," Astrophysical Journal Letters, vol. 523, no. 1, pp. 223-233, 1999.

[60] S. Veeraraghavan and R. D. Davies, "Low frequency galactic backgrounds," in Proceedings of the Particle Physics and Early Universe Conference, Cambridge, UK, April 1997.

[61] F. Boulanger, A. Abergel, J. P. Bernard et al., "The dust/gas correlation at high Galactic latitude," Astronomy \& Astrophysics, vol. 312, no. 1, pp. 256-262, 1996.

[62] T. N. Gautier, T. Cawlfield, and M. W. Werner, "IR emission from small grains in HII regions and reflection nebulae," Bulletin of the American Astronomical Society, vol. 24, p. 1120, 1992.

[63] D. J. Schlegel, D. P. Finkbeiner, and M. Davis, "Maps of dust infrared emission for use in estimation of reddening and cosmic microwave background radiation foregrounds," Astrophysical Journal Letters, vol. 500, no. 2, pp. 525-553, 1998.

[64] K. Rohlfs and T. Wilson, Tools of Radio Astronomy, Springer, 2000.

[65] A. Franceschini, L. Toffolatti, L. Danese, and G. de Zotti, "Discrete source contributions to small-scale anisotropies of the microwave background," Astrophysical Journal, vol. 344, pp. 3545, 1989.

[66] K. M. Górski, A. J. Banday, C. L. Bennett et al., "Power spectrum of primordial inhomogeneity determined from the FOUR-Year COBE DMR sky maps," The Astrophysical Journal Letters, vol. 464, no. 1, pp. L11-L15, 1996.

[67] M. Zaldarriaga, Fluctuations in the cosmic microwavebackground [Ph.D. thesis], Massachusetts Institute of Technology, 1998. 

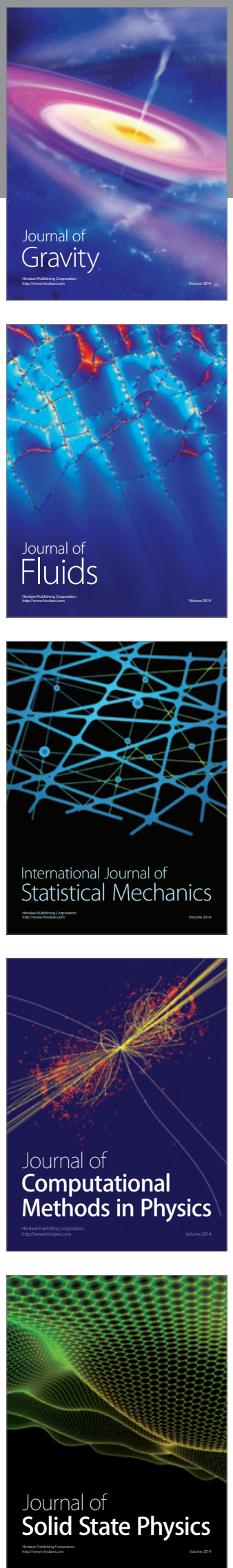

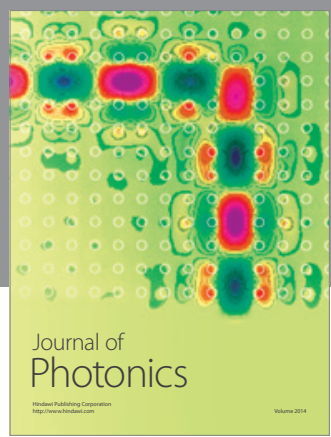

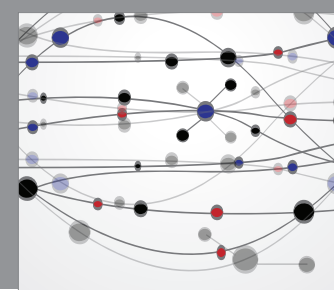

The Scientific World Journal

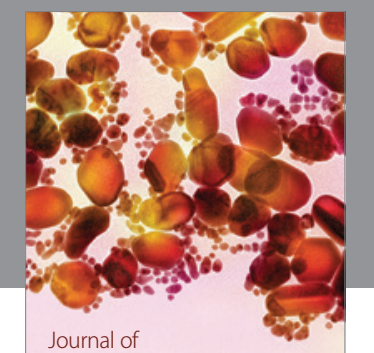

Soft Matter
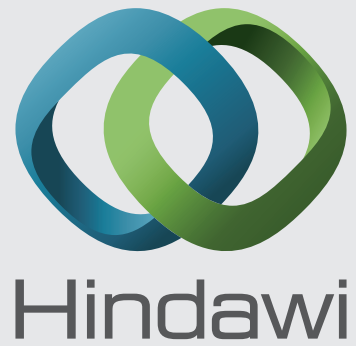

Submit your manuscripts at

http://www.hindawi.com
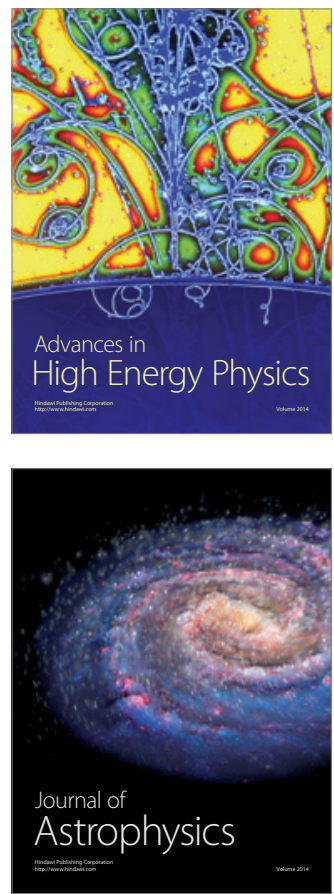
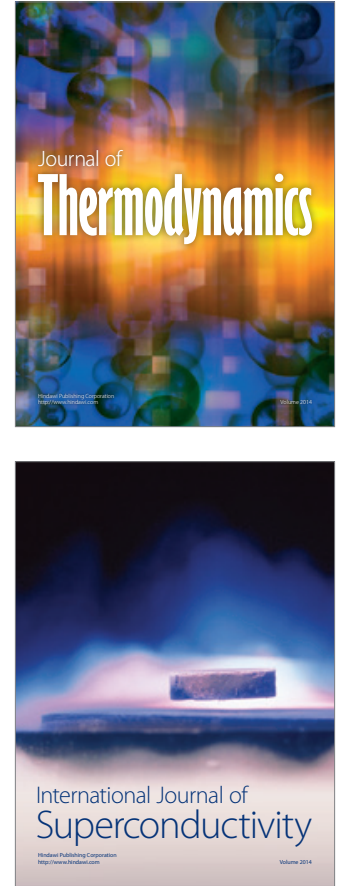
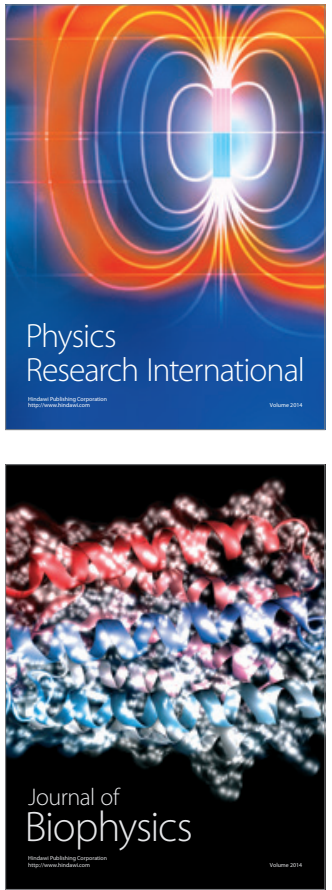
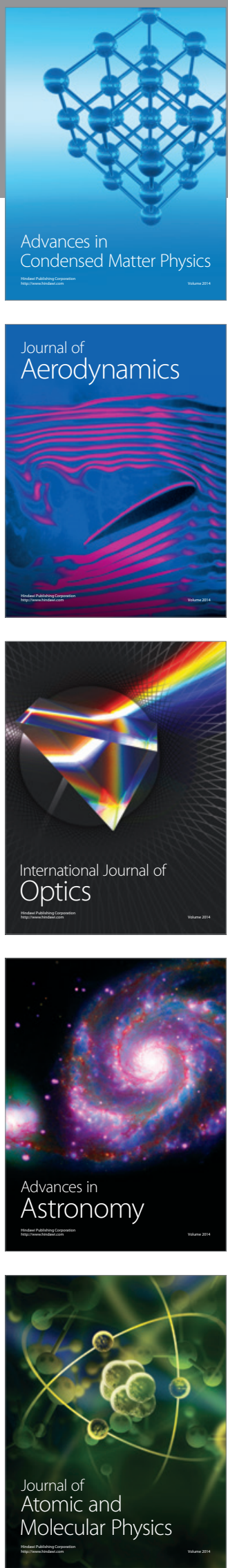